\title{
Osteosclerosis-ichthyosis-premature ovarian failure syndrome
}

INSERM

\section{Source}

INSERM. (1999). Orphanet: an online rare disease and orphan drug data base.

Osteosclerosis-ichthyosis-premature ovarian failure syndrome. ORPHA:75325

This syndrome is characterised by sclerosing bone dysplasia, ichthyosis vulgaris and premature ovarian failure. The bone disorder affects all metaphyseal-diaphyseal regions of the long bones, the skull, and the metacarpals. 